\title{
Distinct lymphocytes subsets in IgM-related neuropathy: clinical-immunological correlations
}

\author{
Raffaele Iorio • Mario Sabatelli • Alessandra Del Grande • Giulia Bisogni • \\ Valentina Damato · Domenico Plantone - Alessandro Marti - Giovanni Frisullo • \\ Angela Romano · Paolo Maria Rossini • Marco Luigetti
}

Received: 4 March 2014/Accepted: 30 August 2014/Published online: 6 September 2014

(C) Springer-Verlag Italia 2014

\begin{abstract}
IgM-related neuropathy generally presents as a late-onset demyelinating polyneuropathy with predominant sensory loss and ataxia. However, we recently reported the clinical, neurophysiological and pathological findings from our cohort and identified in about a third of patients an atypical phenotype. We analyzed by flow cytometry the different lymphocytes subsets in the peripheral blood of patients affected by IgM-related neuropathy, chronic inflammatory demyelinating polyneuropathy (CIDP), monoclonal gammopathy of undetermined significance and healthy subjects, to investigate whether different immunological patterns may differentiate the classical phenotype from atypical forms. IFN-gamma producing CD4+ and CD8+ T lymphocytes, as well as CD4+ and CD8+ T cells expressing T-bet (T-helper type 1, Th1) were increased in CIDP patients. The percentage of circulating CD4+ and CD8 + T cells producing IL-10 as well as the percentage of CD19+ cells expressing Blimp-1 were higher in patients with IgM-neuropathy. We did not find any significant
\end{abstract}

Electronic supplementary material The online version of this article (doi:10.1007/s10072-014-1935-x) contains supplementary material, which is available to authorized users.

R. Iorio

Fondazione Don Gnocchi ONLUS, Milan, Italy

M. Sabatelli · A. Del Grande · G. Bisogni · V. Damato ·

D. Plantone - A. Marti - G. Frisullo · A. Romano ·

P. M. Rossini - M. Luigetti $(\bowtie)$

Department of Geriatrics, Neurosciences and Orthopedics, Institute of Neurology, Catholic University of the Sacred Heart,

Largo Francesco Vito 1, 00168 Rome, Italy

e-mail: mluigetti@gmail.com

P. M. Rossini

IRCCS S. Raffaele-Pisana, Rome and Casa di Cura S. Raffaele

Cassino, Rome, Italy differences in the different lymphocytes subsets in the IgM-related neuropathy between patients with classical and atypical phenotype. Th1 cells are increased in CIDP patients while a T helper type 2-phenotype seems to prevail in patients with IgM-neuropathy. Further studies involving a larger patient population are needed to evaluate if different lymphocytes subset may be involved in different clinical phenotypes of IgM-related neuropathy.

Keywords IgM-related neuropathy · Cytokine . Immunology · Anti-MAG · Clinical phenotype . Neuroscience

\section{Introduction}

Neuropathy associated with IgM monoclonal gammopathy is an immune-mediated disease clinically characterized by symmetrical, distal polyneuropathy with predominant sensory ataxia [1]. Neurophysiological examination typically shows widespread slowing of sensori-motor nerve conduction velocity with prolonged distal latencies [2, 3].

Myelin associated glycoprotein (MAG) specific IgM antibodies, which are present in over $50 \%$ of patients, are thought to have a pathogenic role $[4,5]$. Pathological features of IgM-related neuropathy generally include a variable degree of axonal loss, predominant myelin abnormalities including segmental demyelination, myelin thickening, increased periodicity of myelin lamellae (widening of myelin lamellae, WML) and IgM deposits in myelin sheaths [6].

However, several studies have shown that the pathogenic mechanisms of IgM-related neuropathy are probably heterogeneous and may include a direct pathogenic effect of MAG-IgM [7], endoneural deposition of immunoglobulins 
or light chains [8-10], vasculitis [11], amyloid deposition [12], and infiltration of malignant cells [13]. Furthermore, a recent study demonstrated an increase of interleukin-10 producing lymphocytes in patients with MAG-IgM neuropathy [14].

$\mathrm{T}$ cell lymphocytes can be divided into distinct subsets based on the expression of different transcription factors and the production of different signature cytokines.

Current studies have shown that at least three main functionally different CD4+ effector lymphocytes subpopulations can be distinguished: T-helper (Th) 1 cells characterized by the production of IFN-gamma and the expression of the T-bet transcription factor, Th2 that express GATA-3 and produce IL-10 and IL-4 and Th17 cells that produce IL-17A and express the ROR $\gamma \mathrm{T}$ transcription factor. Moreover, an analog phenotypic distinction has been proposed for the CD8+ effector lymphocytes [15]. The Blimp-1 transcription factor orchestrates the plasma cell differentiation from mature B cells and it is a marker of this lymphocyte subset [16].

Many cases of IgM-related neuropathy with a clinical phenotype not fitting the classic sensory-ataxic form have been described in the literature $[7-12,17,18]$.

We recently reported the clinical, neurophysiological and pathological findings from our cohort of IgM-related neuropathy and we identified in about a third of patients an atypical phenotype [3, 6].

In this study we analyzed the different lymphocytes subsets in patients affected by IgM-related neuropathy to investigate whether distinctive immunological patterns differentiate the classical and the atypical phenotypes.

\section{Patients and methods}

\section{Patients}

Patients were selected from those seen in the Neurology Clinic at our university hospital from 1990 to 2012, on the basis of: (1) clinical and electrophysiological findings suggesting a peripheral neuropathy; and (2) an IgM monoclonal gammopathy.

Patients underwent extensive laboratory studies to rule out other causes of neuropathy, including fasting plasma glucose, glycosylated hemoglobin, $\mathrm{fT}_{3}, \mathrm{fT}_{4}, \mathrm{TSH}$, antithyroid antibodies, serum vitamin $\mathrm{B}_{12}$ and folates, hepatic enzymes, creatinine, urinalysis, antinuclear antibodies (ANA), anti-extractible nuclear antigens (ENA), anti-DNA antibodies, antineutrophil cytoplasmic antibodies (ANCA), circulating $\mathrm{C} 3$ and $\mathrm{C} 4$, and serologic tests for $\mathrm{HBV}, \mathrm{HCV}$ and HIV. All patients underwent immunofixation electrophoresis and hematological evaluation. An enzyme-linked immunosorbent assay (ELISA) was used to test for anti-
MAG antibodies (normal value $<1,000$ Buhlmann titer units); the same test was used in the same laboratory for all patients. We considered positive only patients with high titer of anti-MAG antibodies $(>10,000$ Buhlmann titer units).

Muscle strength was assessed on the MRC (Medical Research Council) scale and severity of neuropathy was evaluated on the Rankin scale.

Based on clinical examination, patients were divided into two phenotypes: classical and atypical phenotype. In the former group, we included those patients in whom clinical manifestations were dominated by signs and symptoms of sensory large fiber dysfunction, such as distal limb paraesthesias, sensory loss and ataxia. Patients with neuropathy not fitting the classic sensory-ataxic form were labeled as having an atypical phenotype [6].

As a control group, we included patients affected by chronic inflammatory demyelinating polyneuropathy (CIDP), by monoclonal gammopathy of undetermined significance (MGUS), and healthy subjects (HS) matched for age and sex.

\section{Nerve conduction studies}

Nerve conduction studies of all subjects were performed using a Key Dantec EMG machine by an EMGer blinded from clinical evaluation using standard techniques [3, 1922]. All studies were performed in a warm room and skin temperature was $>32{ }^{\circ} \mathrm{C}$; if needed, an infrared lamp was used to warm the studied segment.

\section{Isolation of peripheral blood mononuclear cells}

Blood samples were collected at last follow-up for each patient. Peripheral blood mononuclear cells (PBMC) were isolated from venous blood by density gradient centrifugation $(2,500 \mathrm{~g}, 30 \mathrm{~min})$ over a Ficoll-Hypaque density gradient (Pharmacia, Uppsala, Sweden). PBMC were then harvested by pipetting cells from the Ficoll/serum interface and washed twice. The cells were then seeded in RPMI medium supplemented with $10 \%$ fetal calf serum at $1 \times 10^{6}$ cells $/ \mathrm{ml}$ in a 24 -well plate and incubated for $24 \mathrm{~h}$ at $37{ }^{\circ} \mathrm{C}$ in $5 \% \mathrm{CO}_{2}$ in a humidified atmosphere. PBMC were stimulated with anti-CD3 (2 ng/ml; eBioscience) and anti-CD28 (1 ng/ml; eBioscience) antibodies. Brefeldin A was $(10 \mathrm{ng} / \mathrm{ml})$ added in the last $4 \mathrm{~h}$ of incubation for intracellular staining.

Intracellular cytokine analysis

PBMC were washed in phosphate-buffered saline (PBS) and stained with CD4-PyC5 conjugated and CD8-Texas Red (ECD)-conjugated antibodies for $25 \mathrm{~min}$ at $4{ }^{\circ} \mathrm{C}$ in the 
dark. PBMC were then washed, fixed and permeabilized and incubated with the following combination of antibodies: fluorescein isothiocyanate (FITC)-labeled anti-IFNgamma and phycoerythrin (PE)-labeled anti-IL-17; anti-IL9-FITC and anti-IL-10-PE or anti-IL-4-FITC and anti-IL10-PE. Cytometric analysis was performed using a flow cytometer (FC500 cytomics; Beckman-Coulter, Fullerton, California) equipped with a single argon-ion laser operating at $488 \mathrm{~nm}$, interfaced with CXP 21 software. For each analysis at least 50,000 events were acquired and gated on lymphocytes based on forward and side-scatter properties and on CD4 and CD8 expression.

Intracellular transcription factors analysis

PBMC freshly isolated from patients' peripheral blood were incubated with the following surface antibodies: anti-CD8Texas Red (ECD)-conjugated, anti-CD8- phycoerythrincyanine-5 (PC5) conjugated or anti-CD19-PC5 conjugated.

PBMC were then washed, fixed, permeabilized and incubated with antibodies specific for intracellular transcription factors. The following combinations of antibodies were used: PBMC stained with CD4 and CD8 antibodies were incubated with PE-labelled antibodies specific for the transcription factors T-bet, GATA3 or ROR $\gamma \mathrm{T}$ while PBMC stained with CD19 antibody were incubated with Blimp-1-PE antibody.

\section{Statistical analysis}

Fisher's exact test was used for comparing frequencies. Pearson's test was used for correlation analysis. All measurements were expressed as a mean \pm standard deviation. Because the distribution of values was not always normal (assessed through Kolmogorov-Smirnov test), non-parametric analysis (test $U$ Mann-Whitney) was performed. Two-way ANOVA with Tukey's multiple comparisons test was employed to calculate differences between groups. Significance level was set at $p<0.05$.

\section{Results}

Patients

Sixteen patients with IgM neuropathy were included in the study. Thirteen HS, four patients with CIDP and four patients with MGUS served as controls. Extensive laboratories evaluations were unremarkable in all patients. No patient was treated with steroids or immunosuppressant drugs at the time of study enrollment. Patients affected by CIDP were treated with monthly infusions of intravenous immunoglobulin (IVIG). In this group of patients, blood sample was collected just before to start the IVIG infusion.

The classical phenotype was observed in 11 of 16 patients $(69 \%)$ while an atypical phenotype was identified in the remaining five cases $(31 \%)$.

There was no significant difference in the mean age at onset or gender distribution between the two groups, while the disease duration was significantly longer in patients with classical phenotype (124.2 \pm 8.22 vs $108.0 \pm$ 15.26 months, $p=0.0142$ ).

Mean value of Rankin scale was higher in patients with atypical phenotypes compared with patients with the classical form $(2.4 \pm 1.14$ vs $1.09 \pm 0.30, p=0.0025)$.

Muscle strength of tibialis anterior (TA) was significantly reduced in patients with atypical phenotypes if compared with classical forms (mean MRC value $3.2 \pm 1.30$ vs $4.81 \pm 0.40$ for TA, $p=0.0017$ ); no significant difference was observed regarding strength of first digiti interosseous (FDI).

Main clinical, demographic and laboratory characteristics of patients are summarized in supplementary Table S1.

Nerve conduction studies

All patients with classical phenotype showed a demyelinating sensory-motor polyneuropathy [22].

In the group with atypical phenotype, four patients showed a demyelinating neuropathy; the remaining case disclosed a predominantly axonal neuropathy. Main neurophysiological findings of patients are shown in supplementary Table S1.

Immunological data

\section{Intracellular cytokines}

We observed an increase of both CD4+ and CD8 + T cells producing IFN-gamma in patients with CIDP compared with patients with IgM-neuropathy $(p=0.0002$ and $p=0.0006)$, MGUS ( $p=0.019$ and $p=0.0104)$ and HS $(p \leq 0.0001$ and $p=0.0038)$. Both CD4+ and CD8+ IL10 producing $\mathrm{T}$ lymphocytes were increased in patients with IgM-neuropathy compared with HS (both $p \leq$ $0.0001)$, CIDP $(p \leq 0.0001, p=0.0011)$ and MGUS patients ( $p \leq 0.0001$ and $p=0.0006$ ) (Fig. 1).

When we evaluated each subgroup of patients, we confirmed that the percentage of CD4+ and CD8+ producing IFN-gamma was higher in CIDP patients compared with IgM-neuropathy patients with both classical (CD4+, $p=0.0005 ; \mathrm{CD} 8+, p=0.0039)$ and atypical phenotypes (CD4+, $p=0.0081 ; \mathrm{CD} 8+, p \leq 0.0001$ ) (Fig. 2).

Moreover, CD4+ and CD8 $+\mathrm{T}$ cells producing IL-10 were increased in both $\operatorname{IgM}$ neuropathy patients with 
Fig. 1 Intracellular cytokine analysis in CD4+ and CD8+ lymphocytes isolated from the peripheral blood of patients with IgM neuropathy, CIDP,

MGUS, and healthy subjects (HS). After stimulation with anti-CD3 and anti-CD28 IFNgamma producing CD4+ (a) and CD8+ (c) are increased in CIDP patients while IL-10 producing CD4+ (a) and CD8+ (c) lymphocytes are increased in patients with IgM neuropathy. Representative dot plots showing CD4+ IL$10+$ (b) lymphocytes and CD8+ IL-10+ (d) lymphocytes in patients with $\operatorname{IgM}$ neuropathy and in healthy subjects.

$* * p<0.0001 ; * p<0.005$
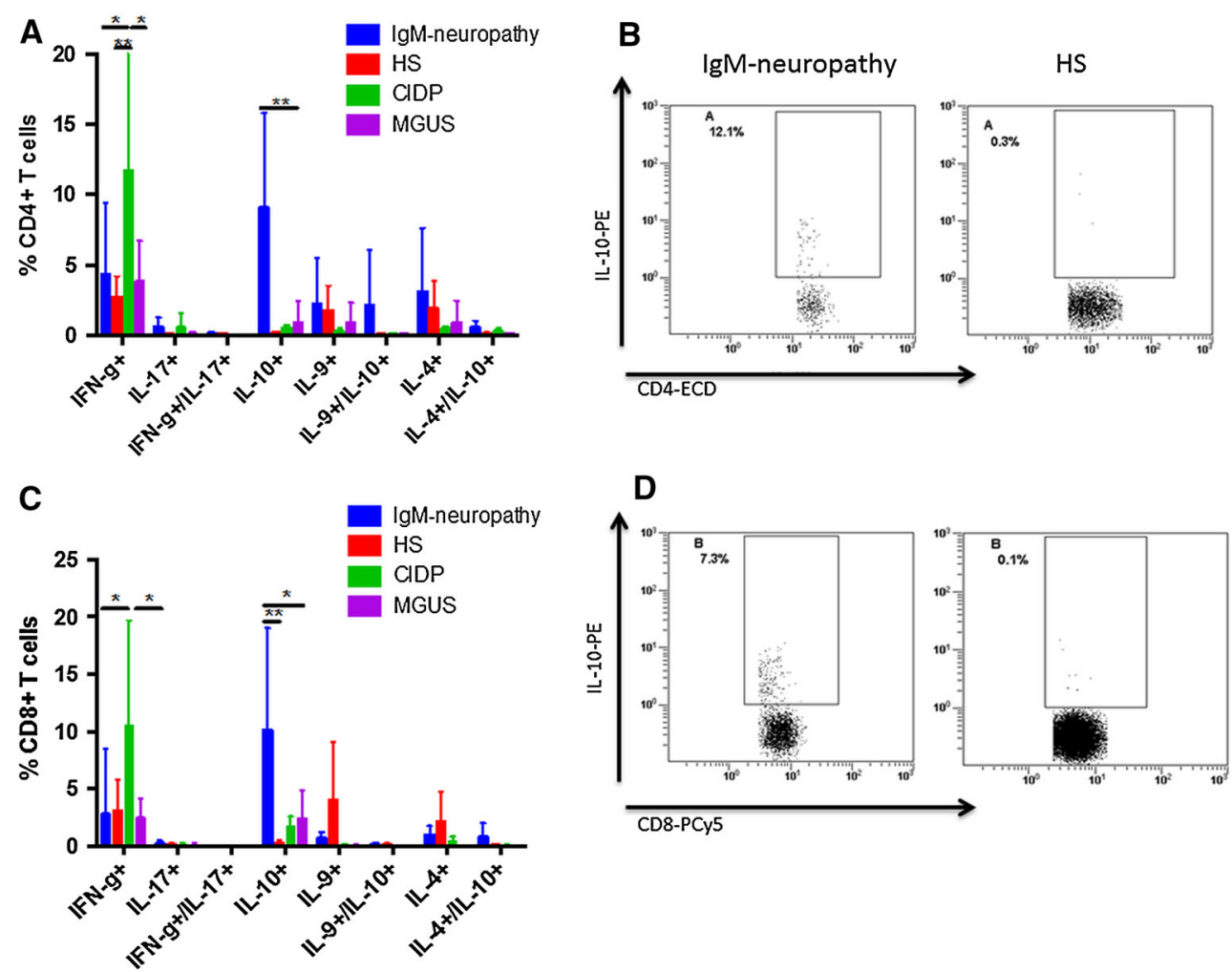

classical and atypical phenotypes compared with HS (CD4+, $p \leq 0.0001$; CD8+, $p \leq 0.0001$ and CD4+, $p \leq$ 0.0001; CD8+, $p \leq 0.0001$, respectively), CIDP (CD4+, $p=0.0006 ; \mathrm{CD} 8+, p=0.0007$ and $\mathrm{CD} 4+, p=0.0007$; CD8+, $p=0.0005$, respectively) and MGUS (CD4+, $p=0.0002 ; \mathrm{CD} 8+, p=0.0005$ and $\mathrm{CD} 4+, p=0.0003$; $\mathrm{CD} 8+, p=0.0005$, respectively) patients (Fig. 2).

\section{Transcription factors}

The analysis of transcription factors expression revealed that the percentage of circulating CD4+ and CD8 $+\mathrm{T}$ cells expressing T-bet was higher in CIDP and MGUS patients compared with patients with IgM-neuropathy (all $p \leq$ 0.0001 ) and HS (all $p \leq 0.0001$ ). Similar results were obtained when we divided IgM-neuropathy patients according to the different phenotypes.

Moreover, the percentage of Blimp-1 expressing CD19+ B lymphocytes (plasmacells) was increased in the total population of IgM-neuropathy patients compared with HS $(p \leq 0.0001)$, CIDP $(p=0.0002)$ and MGUS ( $p$ $=0.0003$ ) patients (Fig. 3a). However, the analysis of Blimp-1 in the two subgroups showed the increase of Blimp-1 expressing CD19+ B lymphocytes only in patients with classical phenotype if compared with HS ( $p \leq$ $0.0001)$, CIDP $(p=0.0001)$ and MGUS $(p \leq 0.0001)$ patients (Fig. 3b).

\section{Discussion}

The typical presentation of neuropathy associated with IgM monoclonal gammopathy is generally characterized by symmetrical, distal polyneuropathy with predominant sensory ataxia and a characteristic electrophysiological pattern [1].

MAG-IgM antibodies, which are present in over $50 \%$ of patients, are generally considered the main causative factor of this demyelinating neuropathy [5]. In a minority of patients, mononeuritis multiplex has been described in which focal nerve involvement may be explained by either vasculitis of the vasa nervorum or immunoglobulin deposition [6]. These patients are classified as having an atypical phenotype. In this study, we observed an increase in different lymphocytes subsets in the peripheral blood of patients with IgM-related neuropathy compared with a control population.

An increase of IL-10 expression in both CD4+ and $\mathrm{CD} 8+\mathrm{T}$ lymphocytes was observed in all patients with IgM-related neuropathy, while patients with CIDP showed an increase of CD4+ and CD8+ T cells expressing T-bet and producing IFN-gamma. These findings are in line with a previous study showing an increase of IL-10 in anti-MAG neuropathy when compared with CIDP [14] and also with a study, performed by our group, showing an increase of circulating CD4+ $\mathrm{T}$ cells producing T-bet in CIDP patients [23]. 

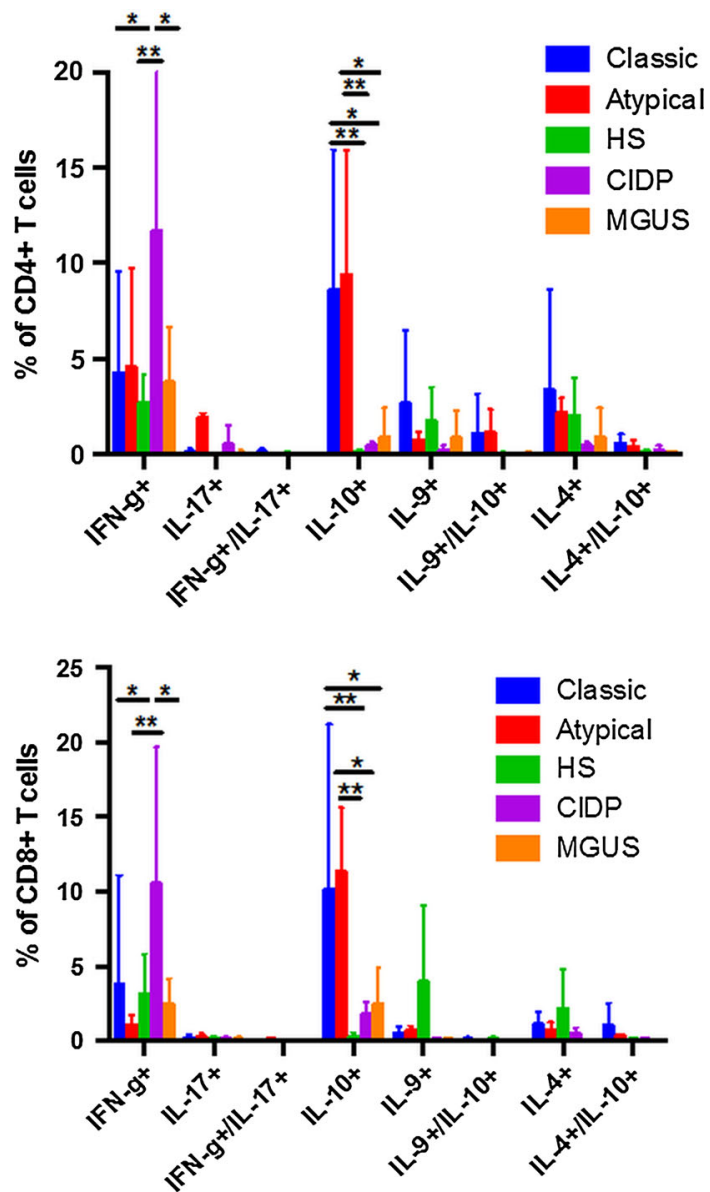

Fig. 2 Intracellular cytokine analysis in CD4+ and CD8+ lymphocytes isolated from the peripheral blood of IgM neuropathy patients with the classical and the atypical phenotype, CIDP patients, MGUS patients, and healthy subjects (HS). After stimulation with anti-CD3 and anti-CD28 IFN-gamma producing CD4+ (upper panel) and CD8+ (lower panel) are increased in CIDP patients compared with IgM neuropathy patients with both phenotypes while IL-10 producing CD4+ (upper panel) and CD8+ (lower panel) lymphocytes are increased in patients with both phenotypes of IgM neuropathy. $* * p<0.0001 ; * p<0.005$

Moreover, the increase of CD19+ cells expressing Blimp-1, namely antibody-producing cells, observed only in IgM-neuropathy patients with the classical phenotype, may explain the observation that patients with the classical phenotype generally improve after monoclonal antibody therapy specific for CD20 (Rituximab), while cases with atypical presentation often show good response to longterm corticosteroids therapy [6].

The better response to Rituximab therapy in patients with the classical phenotype may be explained by the underlying Th2-driven immunity leading to increased CD20-expressing B cell survival and proliferation.

We did not find any significant differences in the cytokines or transcription factors expression in circulating CD4+ and CD8 + T cells between IgM-neuropathy patients with the classical and the atypical phenotypes. This finding may be due to the small size of the population studied.
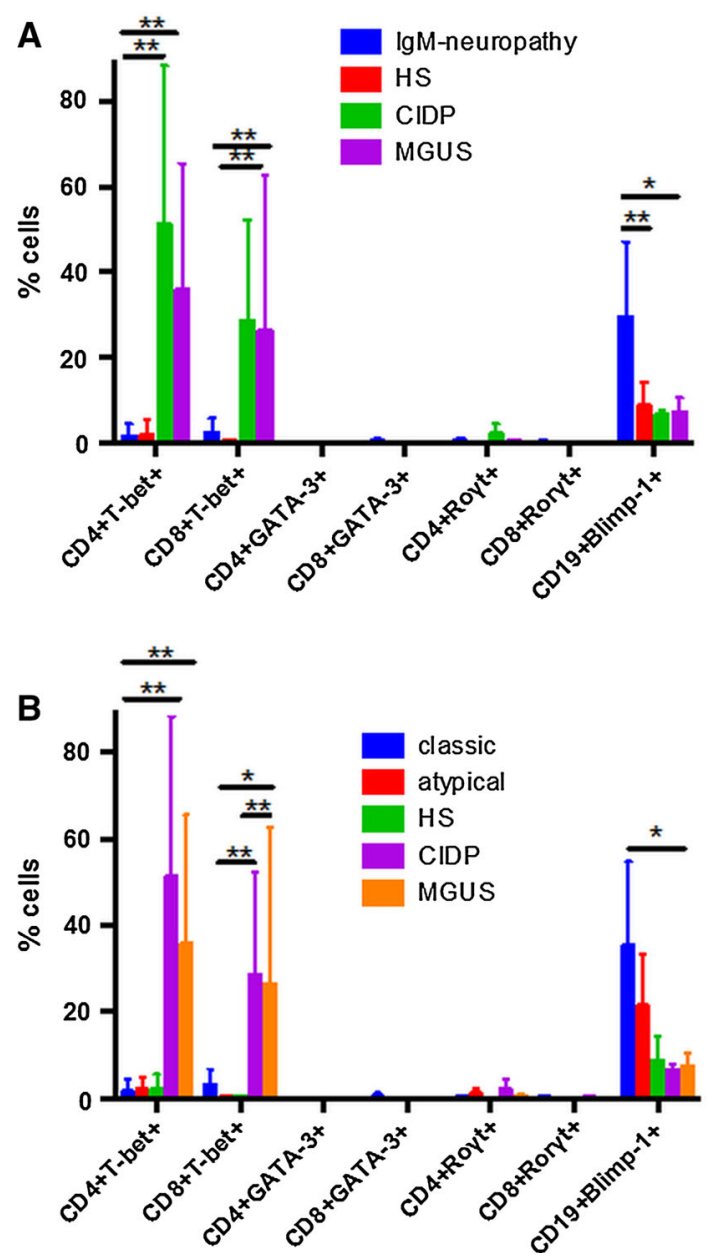

Fig. 3 Transcription factors analysis in CD4+, CD8+ and CD19+ lymphocytes isolated from the peripheral blood of IgM neuropathy patients with the classical and the atypical phenotype, CIDP patients, MGUS patients, and healthy subjects (HS). The percentage of CD4+ and CD8+ $\mathrm{T}$ cells expressing T-bet is higher in CIDP and MGUS patients (a, b). CD19+ B cells expressing Blimp-1 are increased in IgM neuropathy (a) patients, in particular in those with the classical phenotype (b). **p $<0.0001 ; * p<0.005$

"Population-based" studies are needed to better ascertain the role of the different lymphocytes subsets in the course of IgM neuropathy.

Acknowledgments We thank the patients for their participation in this study. This study was not funded.

Conflict of interest The authors declare no competing interest.

\section{References}

1. Nobile-Orazio E, Meucci N, Baldini L, Di Troia A, Scarlato G (2000) Long-term prognosis of neuropathy associated with antiMAG IgM M-proteins and its relationship to immune therapies. Brain 123:710-717 
2. Trojaborg W, Hays AP, Van Den Berg L, Younger DS, Latov N (1995) Motor conduction parameters in neuropathies associated with anti-MAG antibodies and other types of demyelinating and axonal neuropathies. Muscle Nerve 18:730-735

3. Luigetti M, Padua L, Mazza S, Rossini PM, Sabatelli M, Lo Monaco M (2013) Clinical-neurophysiological correlations in a series of patients with IgM-related neuropathy. Clin Neurophysiol 124:1899-1903

4. Nobile-Orazio E, Barbieri S, Baldini L, Marmiroli P, Carpo M, Premoselli S et al (1992) Peripheral neuropathy in monoclonal gammopathy of undetermined significance: prevalence and immunopathogenetic studies. Acta Neurol Scand 85:383-390

5. Hafler DA, Johnson D, Kelly JJ, Panitch H, Kyle R, Weiner HL (1986) Monoclonal gammopathy and neuropathy: myelin-associated glycoprotein reactivity and clinical characteristics. Neurology 36:75-88

6. Luigetti M, Conte A, Montano N, Del Grande A, Madia F, Lo Monaco $\mathrm{M}$ et al (2012) Clinical and pathological heterogeneity in a series of 31 patients with IgM-related neuropathy. J Neurol Sci 319:75-80

7. Baldini L, Nobile-Orazio E, Guffanti A, Barbieri S, Carpo M, Cro L et al (1994) Peripheral neuropathy in IgM monoclonal gammopathy and Waldenström's macroglobulinemia: a frequent complication in elderly males with low MAG-reactive serum monoclonal component. Am J Hematol 45:25-31

8. Luigetti M, Frisullo G, Laurenti L, Conte A, Madia F, Profice P et al (2010) Light chain deposition in peripheral nerve as a cause of mononeuritis multiplex in Waldenström's macroglobulinaemia. J Neurol Sci 291:89-91

9. Vital C, Deminière C, Bourgouin B, Lagueny A, David B, Loiseau P (1985) Waldenström's macroglobulinemia and peripheral neuropathy: deposition of M-component and kappa light chain in the endoneurium. Neurology 35:603-606

10. Grassi MP, Clerici F, Perin C, Borella M, Gendarini A, Quattrini A et al (1998) Light chain deposition disease neuropathy resembling amyloid neuropathy in a multiple myeloma patient. Ital J Neurol Sci 19:229-233

11. Thomas FP, Lovelace RE, Ding XS, Sadiq SA, Petty GW, Sherman WH et al (1992) Vasculitic neuropathy in a patient with cryoglobulinemia and anti-MAG IGM monoclonal gammopathy. Muscle Nerve 15:891-898

12. Bajada S, Mastaglia FL, Fisher A (1980) Amyloid neuropathy and tremor in Waldenström's macroglobulinaemia. Arch Neurol $37: 240-242$
13. Iwashita H, Argyrakis A, Lowitzsch K, Spaar FW (1974) Polyneuropathy in Waldenström's macroglobulinaemia. J Neurol Sci $21: 341-354$

14. Gironi M, Saresella M, Marventano I, Guerini FR, Gatti A, Antonini $\mathrm{G}$ et al (2010) Distinct cytokine patterns associated with different forms of chronic dysimmune neuropathy. Muscle Nerve 42:864-870

15. Nijkamp FP, Pamham MJ (eds) (2011) Principles of immunopharmacology: 3rd revised and extended edition Basel: Birkhäuser Basel

16. Shaffer AL, Lin KI, Kuo TC, Yu X, Hurt EM, Rosenwald A et al (2002) Blimp-1 orchestrates plasma cell differentiation by extinguishing the mature $\mathrm{B}$ cell gene expression program. Immunity 17:51-62

17. Luigetti M, Madia F, Conte A, Tonali P, Sabatelli M (2010) Neuropathy with predominant small fibre involvement associated with abnormal anti-MAG titre. Intern Med 49:2627-2629

18. Kawagashira Y, Kondo N, Atsuta N, Iijima M, Koike H, Katsuno $M$ et al (2010) IgM MGUS anti-MAG neuropathy with predominant muscle weakness and extensive muscle atrophy. Muscle Nerve 42:433-435

19. Kimura J (1989) Electrodiagnosis in disease of the nerve and muscle: principles and practice. FA Davis, Philadelphia, pp 103-138

20. Merolli A, Luigetti M, Modoni A, Masciullo M, Lucia Mereu M, Lo Monaco M (2013) Persistence of abnormal electrophysiological findings after carpal tunnel release. J Reconstr Microsurg 29:511-516

21. Luigetti M, Quaranta D, Modoni A, Mereu ML, Lo Monaco M (2012) Nerve conduction studies of the sural nerve: normative data from a single-center experience. Clin Neurophysiol 123:1891-1892

22. Van den Bergh PY, Hadden RD, Bouche P, Cornblath DR, Hahn A, Illa I et al (2010) European federation of neurological societies/ peripheral nerve society guideline on management of chronic inflammatory demyelinating polyradiculoneuropathy: report of joint task force of the European Federation of Neurological Societies and the Peripheral Nerve Society-first revision. Eur J Neurol 17:356-363

23. Madia F, Frisullo G, Nociti V, Conte A, Luigetti M, Del Grande A et al (2009) pSTAT1, pSTAT3, and T-bet as markers of disease activity in chronic inflammatory demyelinating polyradiculoneuropathy. J Peripher Nerv Syst 14:107-117 